\title{
Study on clinico microbiological profile and antibiotic susceptibility pattern of urinary tract pathogens with Special reference to susceptibility of Escherichia coli to fosfomycin
}

\author{
Raja Sundaramurthy ${ }^{1}$, Rajendran Tiruvanamalai2,", Swetha T. Sivaraman ${ }^{3}$, Ramesh Arunagiri $^{4}$, Jhansi \\ Charles $^{5}$ \\ ${ }^{1,2}$ Assistant Professor, ${ }^{3}$ Student, ${ }^{4}$ Associate Professor, ${ }^{5}$ Professor \& HOD, Dept. of Microbiology, Velammal Medical College \\ Hospital and Research Institute, Madurai, Tamil Nadu, India
}

*Corresponding Author:

Email: rajt1960@gmail.com

\begin{abstract}
Aims: Urinary tract infections (UTI) is the most common infection seeking medical attention. Most of UTI cases were treated empirically with broad-spectrum antibiotics which invariably resulted in development of resistance even to carbapenem. Fosfomycin has broad spectrum of activity which may be useful against these resistant pathogens. Study aims to address microbiological profile, susceptibility pattern of UTI pathogens and fosfomycin susceptibility against all urinary isolates of Escherichia coli (E.coli)

Methodology and Results: All urine samples received in our central laboratory over a period of 2 months (May-June 2017) which showed significant growth were included in the study. After getting the written informed consent, data was collected using a questionnaire regarding demographic and clinical details. In vitro susceptibility of fosfomycin carried out for all E.coli. Among 1280 samples, only $156(12.2 \%)$ yielded significant growth which emphasises the importance of urine culture for definite diagnosis of UTI. Females and $>50$ years of both gender showed higher prevalence. Majority of cases from medicine ward (41.7\%) and ICU $(23.1 \%)$ with diabetes $(55.1 \%)$, catheter $(34.6 \%)$ was common predisposing factor. E.coli $(63.2 \%)$ was the predominant organism followed by Candida (11.6\%) which has become an emerging threat. Higher resistance pattern seen with catheterised group. $40.4 \%$ were extended spectrum beta lactamase (ESBL), 12.8\% inducible AmpC and 29.8\% carbapenamases producers. All E.coli isolates including ESBL, carbapenamase producers showed susceptible to fosfomycin.

Conclusions: Study emphasis evidence based practice is the need of hour to combat with raising resistant pattern. Fosfomycin shown promising results to all ESBL and carbapenamase producing E.coli.
\end{abstract}

Keywords: UTI, Microbiological Profile, Clinical Profile, Fosfomycin.

\section{Introduction}

Urinary tract infections (UTI) - Microbial invasion of urinary tract is the second most common infection in the community, whereas it is the most common hospital acquired infection, accounting for $35 \%$ of all nosocomial infections. ${ }^{1}$ About $10 \%$ of humans develop UTI in some part of their life. ${ }^{2}$ The problem of UTI spans both male and female of all age groups including neonates. ${ }^{3}$ Gender, age, malnutrition, low socioeconomic conditions with poor hygiene, structural and functional abnormalities of urinary tract are few of the main predisposing factors causing UTI. ${ }^{4}$ Escherichia coli, Klebsiella spp., Staphylococcus aureus, Staphylococcus saprophyticus, Proteus spp., Pseudomonas spp., are the most common pathogenic microorganisms isolated in urine. $^{5}$

Most of the UTI cases are treated empirically with broad-spectrum antibiotics over specific antibiotics. Treatment becomes even more challenging in the presence of risk factors such as higher age, comorbidity, and immunosuppression. ${ }^{6}$ Extensive and inappropriate use of antimicrobial agents has invariably resulted in the development of antibiotic resistance which has become a major problem worldwide. ${ }^{7,8}$ Emergence of drug resistance in uropathogens especially Escherichia coli, which is exhibiting high rates of resistance due to the production of extended-spectrum beta-lactamases (ESBLs) and carbapenamases are worrisome which has left us with limited treatment options. ${ }^{9}$

Fosfomycin is a phosphonic acid derivative has a broad spectrum of activity against Gram-positive and Gram-negative bacteria. ${ }^{10}$ Only few studies addressed the invitro activity of fosfomyin against multidrug resistance pathogens. ${ }^{10-12}$ Various studies done worldwide have shown changing patterns in the etiology of UTIs. The present trends of the uropathogens and their susceptibility to various antibiotics including Fosfomycin in all local geographic areas is essential to formulate guidelines for the empirical treatment of UTIs while awaiting the culture sensitivity. ${ }^{5,8}$

The present study aimed to address the common clinical presentation and risk factors for UTI, distribution of pathogenic strains isolated from UTIs and their resistance pattern against commonly used antibiotics and in vitro susceptibility of Fosfomycin against all urinary isolates of Escherichia coli.

\section{Material and Methods}

The study was carried out in a tertiary care hospital for a period of 2 months (May -June 2017) after obtained the approval from the Institutional Ethics Committee. Study was supported by Indian council of Medical 
Research (ICMR) student project (Reference ID: 201707305).

Study included urine samples from all patients received in the central laboratory which showed significant growth in urine culture $\left(>10^{5} \mathrm{CFU} / \mathrm{ml}\right.$ in a clean catch midstream urine; any colony count for supra pubic catheterization (SPC), Percutaneous Nephrostomy (PCN), Cystoscopic specimens) from suspected UTI patients. Probably significant or insignificant growth and mixture of more than 2 organisms even if it is $>10^{5}$ $\mathrm{CFU} / \mathrm{ml}$ were ignored. After getting the written informed consent from the patients showing significant urinary bacterial growth, data was collected using a questionnaire regarding demographic and clinical details and recorded.

\section{Procedure}

Urine was cultured on Cysteine lactose electrolyte deficient media (CLED-Hi media cat no.MP792) for semi quantitative method - For isolation, calibrated $1 \mu \mathrm{L}$ loop with a diameter of $1.3 \mathrm{~mm}$ (Hi media Cat.no:LA023) was used without intermittent heating and incubated at $35-37^{\circ} \mathrm{C}$ for 24 hours. Diagnosis of UTI was confirmed by means of a significant positive urine culture count of $>10^{5}$ colony forming units (CFU) per ml based on Kass concept. Pathogenic organism was further characterised upto species level using various biochemicals wherever applicable.

Antibiotic sensitivity test was done using Kirby Baeur disc diffusion method. Antibiotics were selected according to CLSI 2017 guidelines. ${ }^{[13]}$ The anitbiotic discs selected were Amikacin (30ug), Gentamicin (10ug), Nitrofurantoin (300ug), Ceftriaxone (30ug), Ceftazidime (30ug), Cefepime(30ug), PiperacillinTazobactum (100/10ug), Cotrimoxazole (1.25/23.75ug),Ciprofloxacin (5mg), Imipenam (10ug), Vancomycin (30ug) and Teicoplanin (30ug), Penicillin (10U), Ampicllin(10ug), Linezolid(30ug), Colistin MIC E strip. Antibiotic discs and E strips were bought from Hi-Media diagnostic laboratory, Mumbai. In vitro susceptibility of Fosfomycin (200ug) against all urinary isolates of E.coli was also carried out by disc diffusion methodbased on CLSI 2017 guidelines.

Screening and confirmation of ESBL, Inducible AmpC and Carbapenamase: The screening for extended spectrum beta lactamase (ESBL) production was done using Ceftazidime ( $\leq 22 \mathrm{~mm})$, and Ceftriaxone $(\leq 25 \mathrm{~mm})$. If the organisms showed a zone of inhibition lower than the minimum for the above said antibiotic disc, ESBL positivity was suspected. ${ }^{14}$ If blunting was seen with either Ceftazidime, Ceftriaxone or Piperazillin -Tazobactum against Imipenam, inducible AmpC was suspected and it was confirmed by disc antagonism test. Lawn culture of the test strains made in Muller Hinton agar and disc of Cefoxitin was kept in between Ceftazidime and Ceftriaxone with a gap of $15 \mathrm{~mm}$ from centre to centre of the disc and incubated at $35-37^{\circ} \mathrm{C}$ for 18-24 hours. If blunting was seen between discs, inducible AmpC positivity was confirmed. Carbapenem resistant was suspected if Imipenam disc showing a zone of $\leq 23 \mathrm{~mm}$ and it was confirmed by disc synergy test. Lawn culture of the test strains made in Muller Hinton agar and disc of Imipenam and Imipenam-EDTA discs were kept with a gap of $20 \mathrm{~mm}$ from centre to centre of the disc and incubated at $35-37^{\circ} \mathrm{C}$ for $18-24$ hours. If zone size of Imipenam-EDTA shows $>/-7 \mathrm{~mm}$ excess zone compared to Imipenam then it was confirmed as metallobetalactamase producers.

\section{Statistical Analysis}

The analysis was carried out using the software package SPSS. Age, gender, organisms causing UTI, their antibiotic sensitivity and resistance, symptomatology of these patients and risk factors for UTI were included as variables in the model.

\section{Results}

Clinical Findings: Out of 1280 samples received for urine culture with the clinical diagnosis of UTI, 156 $(12.2 \%)$ samples showed significant growth. Among these, 820 samples were from male patients out of which $85(10.4 \%)$ showed significant growth while in females, out of 460 samples, 71(15.4\%) showed significant growth.

Among those 156 samples which showed significant growth, most common presenting age group was $>50$ years (105/156). Table 1a \&1b shows gender with age group correlation among the study populations.

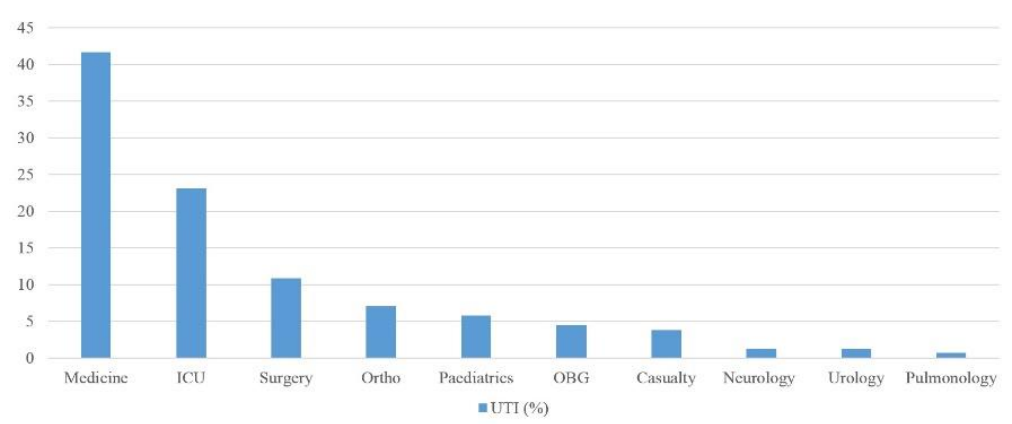

Fig. 1: Ward/Area wise distribution of UTI cases 


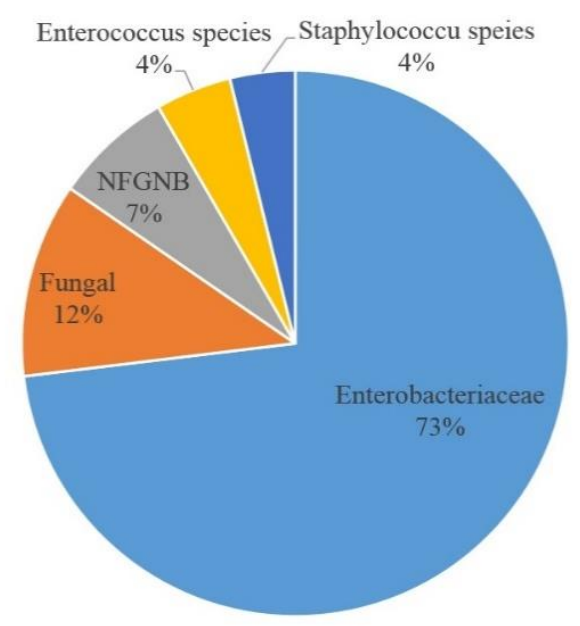

Fig 2: Isolates among the study population

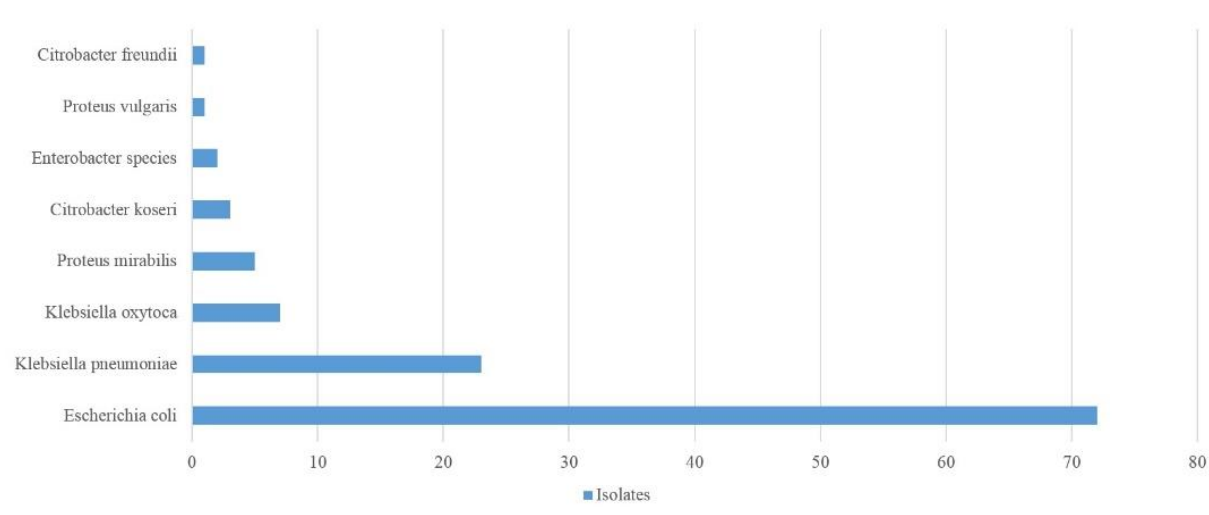

Fig. 3: Isolates among Enterobacteriaceae

UTI was most commonly seen in medicine ward 65(41.7\%) followed by ICU 36(23.1\%). Fig. 1 explains the area wise distribution of UTI cases.

Common presenting symptom was fever, burning micturition, decreased urinary output and lower abdominal pain. Table 2 represents the common presenting symptoms.

\section{Most common presenting symptoms:}

Female-Fever with lower abdominal pain-36.6\% (26/71 cases)

Male-Fever with burning micturition $-44.7 \%$ (38/85)

$>50$ yrs - Burning micturition with decreased urinary output-40\% (42/105)

20-50 yrs- Fever with lower abdominal pain $-78.8 \%$ $(26 / 33)$

$<20$ yrs - Vomiting and increase in frequency $-66.6 \%$ $(12 / 18)$

Diabetes mellitus-55.1\% (86/156 cases) and catheter $34.6 \%$ (54/156 cases) were the common predisposing factors. (Table 3).

Organism isolation:
Gram Negative: 125 isolates $(80.1 \%)$

Enterobacteriaceae- 114 isolates $(73.1 \%)$

Non fermenting Gram negative Bacilli-11 isolates (7\%)

Gram Positive: 13 isolates $(8.3 \%)$

Enterococcus species-7(4.4\%)

Staphylococcus aureus-4(2.6\%)

Coagulase negative Staphylococcus-2(1.3\%)

Fungal: 18 isolates(11.6\%)

Candida species-17(11\%)

Trichosporon-1(0.6\%)

Figure 2 represents the Pathogenic isolates among the study population and

Figure 3 represents the isolates among Enterobacteriaceae.

Antibiotic susceptibility among the isolates: Among Gram negative isolates Cephalosporin showed $81.6 \%$ (93/114) resistant; Fluoroquinolones were the least active drug (98/125=80.7\% resistance) against gram negative uropathogens followed by Cotrimoxazole $(70 / 116=60.3 \%$ resistant $)$ and Aminoglycosides [(Amikacin 66/125= 52.8\%);Gentamicin 71/125=56.8\%)]. Nitrofurantoin 
showed sensitivity among $79 \%$ of our gram negative isolates. Fosfomycin was sensitive among all the isolates of E.coli. Table 4 represents the Gram negative isolates resistant panel.

Among the Gram positive isolates more resistance was seen with Penicillin, Fluoroquinolones $(11 / 13=84.6 \%) \quad$ and Aminoglycosides(10/13=76.9\%). Nitrofurantoin showed $77 \%$ sensitivity. All gram positive isolates were sensitive for Vancomycin, Teicoplanin and Linezolid. Table 5 represents the Gram positive isolates resistant panel.

$40.4 \%(46 / 114)$ Enterobacteriaceae were ESBL producers; $12.8 \%(16 / 125)$ were inducible AmpC producers and $29.8 \%$ (34/114) were Carbapenem resistant isolates. Table 6 represents ESBL, AmpC, CRE among gram negative isolates.

Table 1a: Gender with age group correlation among the study populations

\begin{tabular}{|l|c|c|c|}
\hline Age (Years) & Male & Female & Total \\
\hline$<1$ & 1 & 2 & 3 \\
\hline $1-10$ & 5 & 2 & 7 \\
\hline $11-20$ & 6 & 2 & 8 \\
\hline $21-30$ & 2 & 6 & 8 \\
\hline $31-40$ & 4 & 6 & 10 \\
\hline $41-50$ & 9 & 6 & 15 \\
\hline $51-60$ & 13 & 15 & 28 \\
\hline $61-70$ & 31 & 22 & 53 \\
\hline $71-80$ & 12 & 8 & 20 \\
\hline $81-90$ & 2 & 2 & 4 \\
\hline Total & 85 & 71 & 156 \\
\hline
\end{tabular}

Table 1b: Statistical analysis- Gender with age group correlation among the study populations

\begin{tabular}{|l|c|c|c|}
\hline $\begin{array}{l}\text { Age } \\
\text { (Years) }\end{array}$ & $\begin{array}{c}\text { Male } \\
\mathbf{n = 8 5}(\boldsymbol{\%})\end{array}$ & $\begin{array}{c}\text { Female } \\
\mathbf{n = 7 1}(\boldsymbol{\%})\end{array}$ & $\begin{array}{c}\text { Total } \\
\mathbf{n = 1 5 6}(\boldsymbol{\%})\end{array}$ \\
\hline $0-20$ & $12(14.1)$ & $6(8.4)$ & $18(11.5)$ \\
\hline $21-50$ & $15(17.6)$ & $18(25.4)$ & $33(21.2)$ \\
\hline
\end{tabular}

$>50$

$$
\begin{array}{l|l|l}
58(68.3) & 47(66.2) & 105(67.3) \\
\hline
\end{array}
$$

Chi square statistic is 2.1863. $\mathrm{p}$ value is 0.658915 .

Result is not significant at $\mathrm{p}<0.0$

Table 2: Common Presenting Symptoms among the study population

\begin{tabular}{|l|c|}
\hline \multicolumn{1}{|c|}{ Symptoms } & Number \\
\hline Fever & 63 \\
\hline Burning Micturition & 44 \\
\hline Lower Abdominal Pain & 32 \\
\hline Decreased Urinary Output & 32 \\
\hline Vomiting & 30 \\
\hline Increased Frequency & 24 \\
\hline Loin Pain & 8 \\
\hline Blood in Urine & 3 \\
\hline Pus in urine & 2 \\
\hline
\end{tabular}

Table 3: Predisposing factors for UTI among the study population

\begin{tabular}{|l|c|}
\hline \multicolumn{1}{|c|}{ Predisposing factor } & Number \\
\hline Diabetes alone & 40 \\
\hline Catheter alone & 26 \\
\hline Diabetes + Catheter & 22 \\
\hline Prostatic enlargement alone & 24 \\
\hline Diabetes + Prostatic enlargement & 20 \\
\hline Calculi alone & 1 \\
\hline Calculi + Catheter & 1 \\
\hline Calculi + Diabetes & 1 \\
\hline Calculi + Diabetes + Catheter & 2 \\
\hline Neurogenic bladder alone & 2 \\
\hline Neurogenic bladder + Catheter & 1 \\
\hline Neurogenic bladder + Catheter+ & 2 \\
Calculi & 2 \\
\hline Stricture alone & 1 \\
\hline Stricture + Diabetes & 1 \\
\hline Phimosis & 2 \\
\hline Pregnancy & 6 \\
\hline No specific factors & \\
\hline
\end{tabular}

Table 4: Gram negative isolates resistance panel

\begin{tabular}{|c|c|c|c|c|c|c|c|c|c|c|c|c|}
\hline $\begin{array}{c}\text { Isolate }(n=15 \\
6) \\
\end{array}$ & CAZ & CTR & CPM & CIP & COT & $\mathbf{A K}$ & $\mathbf{G}$ & NIT & PIT & IPM & $\mathbf{C L}$ & FO \\
\hline \multicolumn{13}{|c|}{ Enterobacteriaceae } \\
\hline $\begin{array}{l}\text { Escherichia } \\
\operatorname{coli}(72)\end{array}$ & 59 & 60 & 60 & 59 & 38 & 37 & 41 & 5 & 20 & 12 & 0 & 0 \\
\hline $\begin{array}{l}\text { Klebsiella } \\
\text { pneumoniae( } \\
23)\end{array}$ & 19 & 19 & 19 & 19 & 16 & 15 & 15 & 14 & 14 & 12 & 0 & \\
\hline $\begin{array}{l}\text { Klebsiella } \\
\text { oxytoca }(7)\end{array}$ & 7 & 7 & 6 & 6 & 6 & 5 & 6 & 3 & 6 & 2 & 0 & \\
\hline $\begin{array}{l}\text { Proteus } \\
\text { mirabilis(5) }\end{array}$ & 3 & 5 & 5 & 1 & 2 & 1 & 1 & - & 0 & 0 & - & \\
\hline $\begin{array}{l}\text { Citrobacter } \\
\text { koseri(3) }\end{array}$ & 3 & 3 & 3 & 3 & 3 & 3 & 3 & 3 & 3 & 3 & 0 & \\
\hline
\end{tabular}




\begin{tabular}{|c|c|c|c|c|c|c|c|c|c|c|c|}
\hline $\begin{array}{l}\text { Enterobacter } \\
\text { species(2) }\end{array}$ & 2 & 2 & 2 & 2 & 2 & 2 & 2 & 2 & 2 & 2 & 0 \\
\hline $\begin{array}{l}\text { Proteus } \\
\text { vulgaris }(1)\end{array}$ & 1 & 1 & 1 & 1 & 1 & 1 & 1 & - & 0 & 0 & - \\
\hline $\begin{array}{l}\text { Citrobacter } \\
\text { freundii( } 1 \text { ) }\end{array}$ & 1 & 1 & 1 & 1 & 1 & 0 & 0 & 0 & 1 & 1 & 0 \\
\hline \multicolumn{12}{|c|}{ Non Fermenting Gram Negative Bacilli (NFGNB) } \\
\hline $\begin{array}{l}\text { Pseudomona } \\
s \\
\text { aeruginosa }(9 \\
\text { ) }\end{array}$ & 4 & - & 4 & 2 & - & 1 & 1 & - & 2 & 2 & 0 \\
\hline $\begin{array}{l}\text { Acinetobacte } \\
r \\
\text { Species(2) }\end{array}$ & 2 & 2 & 2 & 2 & 1 & 1 & 1 & - & 2 & 2 & 2 \\
\hline
\end{tabular}

CAZ-Ceftazidime; CTR-Ceftriaxone; CPM-Cefepime; CIP-Ciprofloxacin; COT-Cotrimoxazole; AK-Amikacin; GGentamicin; NIT-Nitrofurantoin; PIT-Piperacillin/Tazobactam; IPM-Imipenem; CL-Colistin; FO-Fosfomycin

Table 5: Gram Positive isolates RESISTANCE panel

\begin{tabular}{|l|c|c|c|c|c|c|c|c|c|c|c|}
\hline \multicolumn{1}{|c|}{ Isolate } & P & A & CIP & G & DO & COT & NIT & VA & TEI & LZ & CX \\
\hline Enterococcus faecium(5) & 4 & 4 & 4 & $\begin{array}{c}5 \\
\text { (HLG) }\end{array}$ & 2 & - & 2 & 0 & 0 & 0 & - \\
\hline Enterococcus faecalis (2) & 1 & 1 & 2 & $\begin{array}{c}2 \\
\text { (HLG) }\end{array}$ & 1 & - & 0 & 0 & 0 & 0 & - \\
\hline Staphylococcus aureus(4) & 4 & - & 4 & 2 & 2 & 2 & 1 & 0 & 0 & 0 & 3 \\
\hline $\begin{array}{l}\text { Coagulase negative } \\
\text { Staphylococcus(2) }\end{array}$ & 2 & - & 1 & 1 & 1 & 1 & 1 & 0 & 0 & 0 & 2 \\
\hline
\end{tabular}

P-Penicillin; A-Ampicillin; CIP-Ciprofloxacin; G-Gentamicin; DO-Doxycyclin; COT-Cotrimoxazole; NITNitrofurantoin; VA-Vancomycin; TEI-Teicoplanin; LZ-Linezolid; CX-Cloxacillin

Table 6: ESBL, AmpC, CRE among gram negative isolates

\begin{tabular}{|l|c|c|c|}
\hline & ESBL producers & Amp C beta lactamases & CRE \\
\hline Escherichia coli(72) & 39 & 8 & 12 \\
\hline Klebsiellapneumoniae(23) & 5 & 2 & 12 \\
\hline Klebsiellaoxytoca(7) & 1 & 1 & 5 \\
\hline Proteus mirabilis(5) & - & - & - \\
\hline Citrobacter koseri(3) & - & - & 3 \\
\hline Enterobacter species(2) & - & 1 & 1 \\
\hline Proteus vulgaris(1) & 1 & - & - \\
\hline Citrobacter freundii(1) & - & - & 1 \\
\hline Total & 46 & 12 & 34 \\
\hline
\end{tabular}

ESBL-Extended Spectrum Beta Lactamases; CRE-Carbapenem Resistant Enterobacteriaceae. AmpC-Inducible AmpC

\section{Discussion}

For the appropriate empirical therapy of UTI, knowledge about present trends of the uropathogens and their susceptibility to various antibiotics in a local geographic area is essential as studies have shown that patterns of etiology and their susceptibility of UTI have shown changing trends over different periods of time from different places. ${ }^{9,15-21}$ Though bacterial infection of the urinary tract is one of the most common cause for seeking medical attention in developing countries, only 156 of 1280 samples $(12.2 \%)$ received in our lab with clinically suspected UTI yielded the significant growth. This emphasises the importance of urine culture for a definite diagnosis of UTI which will avoid unnecessary use of antibiotics.

Our findings revealed that female patients showed higher prevalence $15.4 \%(71 / 460)$ compared to that of male patients $10.4 \%(85 / 820)$ which was in agreement with earlier studies. ${ }^{19-21,23}$ Short urethra, close proximity of female urethral meatus to anus have been reported as factors which influence the higher prevalence in women. Age group analysis revealed that $>50$ years $(105 / 156)$ was the most common presenting age group in both males 58(68.3\%) and females $47(66.2 \%)$. Our finding is in concordance with Sood et al. ${ }^{19}$ who also reported that elderly patients had high prevalence $(41.8 \%)$ and in discordance with other studies. ${ }^{22,24-27}$ This is probably 
because with advancing age, the incidence of UTI increases among males due to prostate enlargement and neurogenic bladder and in females due to urethral stricture and stone.

In our study, majority of cases were from medicine ward (41.7\%), ICU (23.1\%), and post-operative surgical ward (10.9\%) which was similar to the results of Dash $e t$ $a l .{ }^{3}$ who also reported high prevalence of UTI in medicine ward. This may be attributed due to the admission nature of the patients with diabetes and also use of catheter was more common in these premises.

Fever, burning micturition, decreased urinary output and lower abdominal pain were the most common presenting symptoms which is similar to the findings of Little et al., Eshwarappa et al., and Sephai et al. ${ }^{28,8,29}$

Fever with lower abdominal pain was the common presenting complaints in more than $1 / 3 \mathrm{rd}$ of the female patients. In contrast, fever with burning micturition was the common symptom in half of the male patients. Still predictability of UTI by only these symptoms is to be questioned. None of the symptoms individually were potent enough to pick up most of the UTI. Combination of the symptoms has better predictability of UTI. Our findings indicate that clinical presentation plays a minor role in diagnosing UTI and reemphasizing the fact that urine culture is essential to diagnose UTI.

Our study analysis revealed that diabetes mellitus $(55.1 \%)$ and catheter $(34.6 \%)$ were the common predisposing factors which is similar to other studies. ${ }^{[21,22,24,25,27,30]}$ Diabetic nephropathy, reduced immunity contributes to the increased risk for acquiring complicated UTI such as emphysematous pyelonephritis and emphysematous pyelitis among the diabetes patients. Catheter itself is the major predisposing factor for UTI as it favours the infection to spread by biofilm formation on both internal (intraluminal route) and external (periurethral route) catheter surface.

In our study gram negative isolates $(80.1 \%)$ were the most common followed by fungal isolates (11.6\%) which is in concordance with Gupta et al. ${ }^{2}$ Among the Gram negative isolates the bulk was contributed by Enterobacteriaceae $(73.1 \%)$ which is the normal inhabitant of large bowel and non-fermenting gram negative bacilli (NFGNB) contributes 7\%. Escherichia coli $(63.2 \%)$ was the predominant organism among the Enterobacteriaceae followed by Klebsiella pneumoniae $(20.2 \%)$, this proportion of isolates are similar to the study results of Eshwarappa et al., Akram et al., Dash et $a l .8,9,3$

Among the gram positive isolates, Enterococcus species $(4 \%)$ were the common isolates followed by Staphylococcus aureus (3\%) and Coagulase negative Staphylococcus $(1.5 \%)$. Prevalence of Gram positive isolates were not high in our study population which is concordance with the findings of Eshwarappa et al. ${ }^{8}$ but Coagulase negative Staphylococcus(CoNS), previously considered as normal flora, is now a major cause of nosocomial and opportunistic infections which is to be reported cautiously. Both CoNS isolates were associated with catheterised patients.

Among our total 156 isolates 17 were (11.6\%) were Candida species and one was Trichosporon. Candida is the third most common isolate in our set up which is in concordance with the findings of Reshmi C Choudhury et $a l^{30}$ who also reported the higher prevalence of Candiduria (12.3\%) and in discordance with Yashavanth R.et al., and Rahul Kumar Goyal et al., ${ }^{31,32}$ who reported the lower prevalence of candiduria $2.27 \%$ and $2.36 \%$ respectively. All these patients were from ICUs. All the patients with these isolates had both diabetes and catheter as predisposing factors. Candiduria is an emerging threat which was previously assumed as nonpathogenic, now has acquired a greater clinical role in today's scenario of increasing risk factors like diabetes \& catheter, emerging drug resistance and immunocompromised state. Caution has to be taken in reporting candida from urine and it should not be ignored because of risk of candidemia as $31.2 \%$ (5/16 cases) of our cases presented with candidemia.

Antibiotic susceptibility pattern in our study is similar to other studies. ${ }^{23,27}$ Apart from beta lactum group, Fluoroquinolones were the least active drug (98/125 $=80.7 \%$ resistance) against gram negative uropathogens followed by cotrimoxazole $(70 / 116=60.3 \%$ resistant $)$ and aminoglycosides [(Amikacin 66/125= 52.8\%); Gentamicin $71 / 125=56.8 \%)]$. Increasing resistance rate necessitates a regular monitoring for drug resistance in local geographic areas to change the empirical therapy as per the susceptibility pattern. Nitrofurantoin showed some promising results as $79 \%$ of our gram negative isolates were sensitive. Colistin was $100 \%$ sensitive among the gram negative isolates. Among the gram positive isolates other than beat lactum group more resistance was seen with Fluoroquinolones $(11 / 16=84.6 \%) \quad$ and aminoglycosides $\quad(10 / 13=76.9 \%)$. Here also Nitrofurantoin showed some promising results as $77 \%$ of our gram positive isolates were sensitive. All gram positive isolates were sensitive for Vancomycin, Teicoplanin and Linezolid.

In our study, $40.4 \%(46 / 114)$ Enterobacteriaceae were ESBL producers and among Escherichia coli (39/72) $54.2 \%$ were ESBL which is similar to the study results of Eshwarappa et al. ${ }^{8}$, where $52.2 \%$ of the isolates were ESBL-positive. Previous studies in India have reported an ESBL positivity rate between $26.9 \%$ and $48.3 \% \cdot{ }^{[16,23,26,28,30-32]}$ ESBL producers do not respond to the usually prescribed empirical therapy of cephalosporins which will ultimately lead to increased risk of associated morbidity and mortality, and cost of therapy. Presently, alternative antimicrobial therapy to treat ESBL-positive UTI on outpatient basis is limited. Beta lactam with inhibitor combinations and Carbapenems are the most effective in this situation but need to be administered intravenously or - 
intramuscularly. Nitrofurantoin and Fosfomycin which can be given orally were active invitro against $90 \%$ and $100 \%$ of the above mentioned ESBL isolates.

In our study $12.8 \%(16 / 125)$ were inducible AmpC producers. These isolates do not respond to Beta lactum with inhibitor combinations. Carbapenems are the most effective in this situation. Fosfomycin among these resistant isolates also showed $100 \%$ sensitivity invitro against E.coli and $87.5 \%$ of Nitrofurantoin showed sensitivity against these inducible AmpC producers.

In our study $29.8 \%(34 / 114)$ were carbapenem resistant isolates. Among these 52.2\% (12/23) of Klebsiella pneumoniae and 71.4\%(5/7) of Klebsiella oxytoca were carbapenem resistant and $16.7 \%$ (12/72) of E.coli showed carbapenem resistance. The situation is worsening everyday as no new antibiotics against these multidrug-resistant organisms are in advanced stages of clinical development. Even Nitrofurantion showed only $11.8 \%$ (4/34) sensitive against these carbapenem resistant isolates. Fosfomycin showed promising invitro results against the CRE of E.coli.

In our study, all the $E$. coli tested isolates including ESBL producers, Carbapenamase producers were susceptible to fosfomycin invitro. Our study reveals some evidence that fosfomycin might be a promising solution for the treatment of such multi drug resistant infections.

The prevalence of infection by these organisms in our study is comparable to those of other studies from India as well as other countries. ${ }^{23,27}$ The uropathogens identified in our study are similar to those of many other studies conducted in different countries, however different from some study results. ${ }^{21,24}$ The similarities and differences in the type and distribution of uropathogens may result from different environmental conditions and host factors, and practices such as healthcare and education programmes, socioeconomic standards and hygiene practices in each country. Though diabetes was the most predominant predisposing factor, no difference was found among the uropathogenic profile or the antibiotic sensitivity pattern between diabetic and non-diabetic.But the second common predisposing factor (catheterised patients) had higher resistance pattern overall compared to other groups.

\section{Conclusion}

UTI was most common amongfemale patients and $>50$ years of both sex in our population too.

Medicine ward and ICUs had most number of cases due to the admission nature of the patients with diabetes and catheter which is also reported as an importance predisposing factor in our study. Though combination of the symptoms had somewhat better predictability of UTI, culture remains the gold standard. Escherichia coli was the predominant isolate followed by Candida species which emphasis that Candiduria is an emerging threat; similarly Coagulase negative Staphylococcus, previously considered as normal flora, is now a major cause of UTI in catheterised patients. No difference was found among the uropathogenic profile or the antibiotic sensitivity pattern between diabetics and non-diabetics. But catheterised patients had the higher resistance pattern overall compared to other groups. Increasing resistance of ESBL, Inducible AmpC and Carbapenamases is worrisome. In our study, all the $E$. coli tested isolates including ESBL producers, Carbapenamase producers were susceptible to fosfomycin invitro. Our study reveals some evidence that fosfomycin might be a promising solution for the treatment of such infections.

\section{Limitations}

Fosfomycin was tested only against E.coli isolates as disc diffusion guidelines was only available for E.coli as per CLSI. Due to non-affordability, MIC of fosfomycin for other isolates were not carried out. In vivo analysis of fosfomycin use for both complicated and uncomplicated UTI is at present the need of the hour as we are left with only few drugs against CRE isolates.

\section{Implications}

Only $12.2 \%$ of the samples with clinically suspected UTI yielded the significant growth that emphasises that urine culture is essential to avoid unnecessary use of antibiotics. So evidence based practice is always essential.

All the E. coli tested isolates including ESBL producers, Carbapenamase producers were susceptible to fosfomycin invitro. Our study reveals some evidence that fosfomycin might be a promising solution for the treatment of such infections.

As nearly half of the isolates had ESBL, we have formulated the ideal choice for empirical therapy as beta lactum with inhibitor combinations like Cefaperazone Sulbactum for complicated UTI in our set up.

\section{Acknowledgement}

I'm very thankful to our Professor and Head Dr Jhansi Charles who provided all support and encouragements to carry out the work.

\section{Funding}

Project was carried with the support of Indian council of Medical Research (ICMR) student project (Reference ID: 2017-07305).

\section{Conflict of Interest: Nil}

\section{References}

1. Manges AR, Natarajan P, Solberg OD, Dietrich PS, Riley LW.(2006). The changing prevalence of drug-resistant Escherichia coli clonal groups in a community: evidence for community outbreaks of urinary tract infections. Epidemiol Infect. 134(2):425-31.

2. Gupta K, Scholes D, Stamm WE.(1999). Increasing prevalence of antimicrobial resistance among 
uropathogens causing acute uncomplicated cystitis in women. JAMA. 24;281(8):736-8.

3. Dash M, Padhi S, Mohanty I, Panda P, Parida B.(2013). Antimicrobial resistance in pathogens causing urinary tract infections in a rural community of Odisha, India. J Fam Community Med. 20(1):20-6.

4. Niranjan V, Malini A. (2014). Antimicrobial resistance pattern in Escherichia coli causing urinary tract infection among inpatients. Indian J Med Res. 139(6):945-8.

5. Renuart AJ, Goldfarb DM, Mokomane M, Tawanana EO, Narasimhamurthy M, Steenhoff AP. (2013).

Microbiology of Urinary Tract Infections in Gaborone, Botswana. PLOS ONE. 4;8(3):e57776.

6. Arias CA, Murray BE.(2009). Antibiotic-resistant bugs in the 21st century--a clinical super-challenge. N Engl J Med. 29;360(5):439-43.

7. V G. (2002) Antibiotic resistance pattern in uropathogens. Indian J Med Microbiol. 1;20(2):96.

8. Eshwarappa M, Dosegowda R, Aprameya IV, Khan MW, Kumar PS, Kempegowda P.(2011). Clinicomicrobiological profile of urinary tract infection in South India. Indian J Nephrol. 1;21(1):30.

9. Akram M, Shahid M, Khan AU.(2007). Etiology and antibiotic resistance patterns of community-acquired urinary tract infections in J N M C Hospital Aligarh, India. Ann Clin Microbiol Antimicrob. 23;6:4.

10. Neuner EA, Sekeres J, Hall GS, Duin D van. (2012). Experience with Fosfomycin for Treatment of Urinary Tract Infections Due to Multidrug-Resistant Organisms. Antimicrob Agents Chemother. 1;56(11):5744-8.

11. Maraki S, Samonis G, Rafailidis PI, Vouloumanou EK, Mavromanolakis E, Falagas ME. (2009). Susceptibility of Urinary Tract Bacteria to Fosfomycin. Antimicrob Agents Chemother. 1;53(10):4508-10.

12. Falagas ME, Giannopoulou KP, Kokolakis GN, Rafailidis PI. (2008). Fosfomycin: Use Beyond Urinary Tract and Gastrointestinal Infections. Clin Infect Dis. 1;46(7):106977.

13. CLSI Publishes New Antimicrobial Susceptibility Testing Standards - CLSI 2017. Available from: http://clsi.org/blog/2015/01/08/clsi-publishes-newantimicrobial-susceptibility-testing-standards/

14. Picozzi SCM, Casellato S, Rossini M, Paola G, Tejada M, Costa E. (2014). Extended-spectrum beta-lactamasepositive Escherichia coli causing complicated upper urinary tract infection: Urologist should act in time. Urol Ann. 6(2):107-12.

15. Magale Hi, Kassim Ia, Odera Sa, Omolo Mj, Jaoko Wg, Jolly Pe.(2015). Antibiotic Susceptibility Of Organisms Causing Urinary Tract Infection In Patients Presenting At Kenyatta National Hospital, Nairobi. East Afr Med J. 92(7):333-7.

16. Das RN, Chandrashekhar TS, Joshi HS, Gurung M, Shrestha N, Shivananda PG.(2006). Frequency and susceptibility profile of pathogens causing urinary tract infections at a tertiary care hospital in western Nepal. Singapore Med J. 47(4):281-5.

17. Gonzalez CM, Schaeffer AJ.(1999). Treatment of urinary tract infection: what's old, what's new, and what works. World J Urol. 17(6):372-82.

18. Beyene G, Tsegaye W.(2011). Bacterial Uropathogens in Urinary Tract Infection and Antibiotic Susceptibility Pattern in Jimma University Specialized Hospital, Southwest Ethiopia. Ethiop J Health Sci. 21(2):141-6.

19. Sood S, Gupta R.(2012). Antibiotic Resistance Pattern of Community Acquired Uropathogens at a Tertiary Care Hospital in Jaipur, Rajasthan. Indian J Community Med Off Publ Indian Assoc Prev Soc Med. 37(1):39-44.
20. Orrett FA. (2001). Urinary tract infections in general practice in a rural community in South Trinidad. Saudi Med J. 22(6):537-40.

21. Oladeinde BH, Omoregie R, Olley M, Anunibe JA. (2011). Urinary tract infection in a rural community of Nigeria. North Am J Med Sci. 3(2):75-7.

22. Magale Hi, Kassim Ia, Odera Sa, Omolo Mj, Jaoko Wg, Jolly Pe. (2015). Antibiotic Susceptibility Of Organisms Causing Urinary Tract Infection In Patients Presenting At Kenyatta National Hospital, Nairobi. East Afr Med J. 92(7):333-7.

23. Das RN, Chandrashekhar TS, Joshi HS, Gurung M, Shrestha N, Shivananda PG. (2006). Frequency and susceptibility profile of pathogens causing urinary tract infections at a tertiary care hospital in western Nepal. Singapore Med J. 47(4):281-5.

24. Nicolle LE. (2014). Catheter associated urinary tract infections. Antimicrob Resist Infect Control. 25;3:23.

25. Brindha SM, Jayashree M, Singhi S, Taneja N. (2011). tudy of nosocomial urinary tract infections in a pediatric intensive care unit. J Trop Pediatr. 57(5):357-62.

26. Anatoliotaki M, Galanakis E, Schinaki A, Stefanaki S, Mavrokosta M, Tsilimigaki A.(2007). Antimicrobial resistance of urinary tract pathogens in children in Crete, Greece. Scand J Infect Dis. 39(8):671-5.

27. Falagas ME, Giannopoulou KP, Kokolakis GN, Rafailidis PI.(2008). Fosfomycin: Use Beyond Urinary Tract and Gastrointestinal Infections. Clin Infect Dis. 1;46(7):106977.

28. Shaifali I, Gupta U, Mahmood SE, Ahmed J.(2012). Antibiotic Susceptibility Patterns of Urinary Pathogens in Female Outpatients. North Am J Med Sci. 4(4):163-9.

29. Little P, Merriman R, Turner S, Rumsby K, Warner G, Lowes JA. (2010). Presentation, pattern, and natural course of severe symptoms, and role of antibiotics and antibiotic resistance among patients presenting with suspected uncomplicated urinary tract infection in primary care: observational study. BMJ. 5;340:b5633.

30. Sepahi MA, Heidari A, Shajari A. (2010). Clinical manifestations and etiology of renal stones in children less than 14 years age. Saudi J Kidney Dis Transplant. $1 ; 21(1): 181$.

31. Choudhuri JA, Pergamit RF, Chan JD, Schreuder AB, McNamara E, Lynch JB. (2011). An electronic catheterassociated urinary tract infection surveillance tool. Infect Control Hosp Epidemiol. 32(8):757-62.

32. R. Y, M.P. S, U.A. B, R. R, K.B. A.(2013). Candiduria: Prevalence and Trends in Antifungal Susceptibility in A Tertiary Care Hospital of Mangalore. J Clin Diagn Res JCDR. 7(11):2459-61.

33. Goyal R, Sami H, Mishra V, Bareja R, Behara R. (2016). Non-Albicans Candiduria: An Emerging Threat. J Appl Pharm Sci. 048-50. 